\title{
SÍNDROME DO INTESTINO CURTO: UMA NOVA ALTERNATIVA DE TRATAMENTO CIRÚRGICO
}

\author{
Short-bowel syndrome: a new alternative for surgical treatment \\ Orli FRANZON, Hideo SUZUKI, Karina Midori SATO, \\ Maria Claudia PICCOLI, Marília Granzotto VOLPATO
}

Trabalho realizado no Departamento de Cirurgia Geral do Hospital Regional de São José - Dr. Homero de Miranda Gomes (HRSJ), São José, SC, Brasil.

DESCRITORES - Síndrome do intestino curto. Nutrição parenteral total. Cirurgia.
RESUMO - Introdução - A síndrome do intestino curto resulta de comprimento total de intestino delgado inadequado para manter a nutrição, e assim, os pacientes tornam-se cronicamente dependentes de nutrição parenteral total. Numerosas estratégias cirúrgicas foram estudadas para restabelecer a função intestinal normal. Objetivo - Propor a utilização da inversão de segmento de alça associada à STEP - Serial Transverse Enteroplasty - para aumentar a área absortiva intestinal em pacientes com síndrome do intestino curto. Método - Os autores descrevem uma nova alternativa para o tratamento cirúrgico da síndrome do intestino curto, demonstrando sua facilidade e eficiência para o objetivo proposto. Resultados - As duas técnicas associadas permitiram alongar a alça intestinal em 2,6 vezes do comprimento original, com posterior ganho de peso, redução do número de evacuações para uma exoneração ao dia e melhora da qualidade de vida social e psicológica. Conclusão - Essa técnica de inversão de segmento de alça intestinal e posterior STEP surge como tratamento eficiente e definitivo à síndrome do intestino curto de forma promissora e segura permitindo retorno à alimentação por via oral com satisfatória absorção de nutrientes e ganho de peso, retorno da motilidade efetiva intestinal, associada à baixa morbidade e melhoria da qualidade de vida social e psicológica. Apesar de requerer várias cargas de grampeadores lineares apresenta baixa morbidade e passa a tornar-se um potencial substituto para o transplante intestinal.

ABSTRACT - Background - The short bowel syndrome results of inadequate total length of small intestine that is not enough to nutrition, and so, the patients become dependents of total parenteral nutrition. Numerous surgical strategies had been studied to reestablish the normal intestinal function. Objective Consider the inversion segment of small intestine associated with STEP - Serial Transverse Enteroplasty - to increase the intestinal absorptive area in patients with short bowel syndrome. Method - The authors describe a new alternative for surgical treatment of short bowel syndrome, demonstrating it's easiness and efficiency for the considered objective. Results - The two techniques associated, had allowed lengthening the small intestine in 2,6 times the original length, with posterior profit of weight, reduction the number of evacuations. Conclusion This technique of inversion segment of small intestine and posterior STEP is efficient, appears as definitive and promising technique of treatment to short bowel syndrome allowing return to oral feeding, with satisfactory absorption of nutrients, return to normal intestinal movement, associated to low morbidity and improvement the quality of social and psychological life. It presents low morbidity and is a potential substitute to intestinal transplant.
HEADINGS - Short bowel syndrome. Total parenteral nutrition. Surgery.

\section{INTRODUÇÃO}

A síndrome do intestino curto (SIC) resulta de um comprimento total de intestino delgado que é inadequado para manter a nutrição via oral satisfatoriamente. As causas mais frequentes no adulto são doenças malignas, radiação e insuficiência vascular; na criança, enterocolite necrotizante e anormalidades intestinais ${ }^{27}$. Apesar da notável capacidade de adaptação após ressecções extensas este mecanismo adaptativo pode ser sobrepujado se houver mais de $70 \%$ de ressecção ou menos de 200 
cm de intestino delgado 8,14,15,16,28. As consequências fisiopatológicas após ressecção intestinal dependem do comprimento e do local da ressecção20. Pacientes com menos de $100 \mathrm{~cm}$ de intestino delgado, sem o cólon, provavelmente necessitarão de nutrição parenteral para sobreviver. Em contrapartida, $50 \mathrm{~cm}$ de intestino delgado costuma ser suficiente para a nutrição oral adequada se a maioria do cólon estiver presente ${ }^{20}$.

Após ressecção maciça do intestino delgado o tratamento precoce é direcionado para o controle da diarréia, reposição de líquidos e instituição de nutrição parenteral total (NPT). Outras medidas importantes incluem a redução da secreção gástrica com antagonistas do receptor $\mathrm{H} 2$ ou bloqueadores da bomba de prótons, suplementação de potássio, cálcio, zinco e particularmente magnésio de acordo com a monitoração dos níveis séricos ${ }^{1,9,21,22,23,33,34}$. O análogo da somatostatina de ação longa, octreotídeo, parece reduzir a quantidade de diarréia durante a fase inicial da SIC. Outras complicações incluem aumento na incidência de cálculos biliares devido à ruptura da circulação enterohepática e nefrolitíase por hiperoxalúria. Os papéis dos hormônios implicados como agentes tróficos administrados sistematicamente como o hormônio do crescimento, enteroglucagon, fator de crescimento insulina-like e a glutamina estão sendo avaliados com insuficientes evidências clínicas descritas 4,8,14,15,16,20,21,28,31.

Numerosas estratégias cirúrgicas foram tentadas em pacientes cronicamente dependentes de NPT $21,24,25,26,29,30$. Procedimentos para retardar o tempo de trânsito intestinal permitindo tempo adicional de contato para a absorção de nutrientes e líquidos como a construção de válvulas e esfíncteres tem sido descritos, porém com resultados inconsistentes relatados. Outros procedimentos como a interposição colônica, as alças recirculantes de intestino delgado e o marcapasso elétrico retrógrado foram tentados sem sucesso em humanos. Rygick e Nasarov ${ }^{19}$, em 1969, propuseram um método de inversão de segmento ileal de aproximadamente $20 \mathrm{~cm}$ criando um segmento anti-peristáltico de delgado, lentificando a passagem do quimo e aumentando absorção, porém, obtendo como consequência uma dilatação à montante da anastomose proximal. Transplante de intestino delgado é hoje realizado com resultados promissores, mas há insuficiente descrição literária principalmente pela restrição de candidatos ao procedimento ${ }^{5,7,18}$.

A técnica de alongamento intestinal com redução do diâmetro luminal foi primeiramente descrita por Bianchi em $1980^{8}$ com subsequentes relatos de sucesso no manejo cirúrgico da SIC em crianças e neonatos 2,10,17,27,32.

A enteroplastia seriada transversa (Serial Transverse Enteroplasty - STEP) pode suplantar essas limitações no momento em que é tecnicamente mais fácil, não requer anastomose intestinal e adaptase a diferentes níveis de dilatação intestinal6,12,13.
Fundamenta-se em aplicações de grampeadores lineares perpendiculares ao eixo intestinal com sentidos alternados, preservando adequada vascularização em todos os segmentos do intestino. Forma-se, desse modo, um canal intestinal com tamanho determinado pelo cirurgião, de diâmetro menor que o original e significativamente maior em comprimento. A nova técnica neste momento apresentada parte da idéia proposta por Rygick e Nasarov ${ }^{19}$ de inversão de alça (Figura 1) com formação de alça anisoperistáltica somada à STEP da seguinte forma: partindo-se de uma inversão prévia de um segmento de delgado, tem-se uma alça anisoperistáltica com diâmetro dilatado satisfatoriamente. Sequencialmente procedese à reversão da alça anisoperistáltica e à enteroplastia com aplicação intercalada dos grampeadores obtendo aumento significativo do comprimento intestinal, sem prejuízo ao diâmetro luminal. Essa técnica surge como tratamento definitivo à SIC de forma promissora e segura permitindo retorno à alimentação via oral com satisfatória absorção de nutrientes e ganho de peso, retorno da motilidade intestinal associada à baixa morbidade e melhoria da qualidade de vida.

Os autores deste trabalho propõem a utilização da inversão de alça associada à STEP - Serial Transverse Enteroplasty - para aumentar a área absortiva em pacientes que sofreram extensas ressecções intestinais e permanecem cronicamente dependentes de NPT.

\section{MÉTODO}

S.P.B, 45 anos, feminina, branca, natural de Santa Catarina, foi submetida à extensa ressecção de grande parte do jejuno, íleo e ceco por trauma contuso abdominal em fevereiro do ano de 2000, permanecendo com $50 \mathrm{~cm}$ de alça de jejuno remanescente com anastomose jejuno-transverso. Após a operação, passou a apresentar em média 15 exonerações ao dia e dependência de nutrição parenteral total a cada quatro meses.

Em agosto de 2001 foi submetida à rotação de $20 \mathrm{~cm}$ do segmento do jejuno distal com anastomose término-terminal em plano único sero-muscular à Rygick e $\operatorname{Nasarov}^{19}$ (Figura 1). Após quatro dias, foi iniciada dieta via oral exclusiva e, na evolução, passou a apresentar três a quatro exonerações ao dia.

A paciente permaneceu durante quatro anos aguardando a obtenção dos grampeadores. Em dezembro de 2005, foi realizada a reversão da alça anisoperistáltica e aplicação de 25 cargas de grampeadores lineares de $75 \mathrm{~mm}$ à STEP (Figura 2) no segmento dilatado do jejuno à montante e também no segmento que havia sido revertido (Figura 3). Nesse período, o intestino apresentava largura de 8 $\mathrm{cm}$ e a aplicação do grampeador foi realizada à $4 \mathrm{~cm}$ de largura da borda e à $2 \mathrm{~cm}$ de distância entre eles. Dessa forma, a largura intestinal reduziu de $8 \mathrm{~cm}$ para 
$4 \mathrm{~cm}$, e o comprimento, de $50 \mathrm{~cm}$ para $180 \mathrm{~cm}$. Foi iniciada a dieta via oral exclusiva no terceiro dia do pósoperatório. Após dois meses, apresentava albumina de 3,9g/dL, ferritina $1256 \mathrm{ng} / \mathrm{dL}, \mathrm{Hb} 13.4 \mathrm{~g} / \mathrm{dL}, \mathrm{Ht}$ 40\%, leucócitos de $8.200 \mathrm{~mm}^{3}$, triglicerídeos de $187 \mathrm{mg} / \mathrm{dL}$, vit B12 de $187 \mathrm{pg} / \mathrm{mL}$. A paciente apresentou melhora clínica importante com diminuição da frequência de evacuações para uma vez ao dia, ganho de peso de aproximadamente $20 \mathrm{Kg}$ em 12 meses, melhora da nutrição e da qualidade de vida.

\section{TÉCNICA}

Um segmento de $20 \mathrm{~cm}$ de jejuno distal é seccionado e seu sentido invertido de forma a confeccionar uma alça anisoperistáltica, conforme descrição de Rygick e Nasarov ${ }^{19}$ (Figura 1) e o trânsito intestinal é refeito com anastomose términoterminal em plano único sero-muscular com fio PDS ${ }^{\circledR}$ (polidioxanone) 3-0. Após a anastomose intestinal, o meso é suturado, com pontos separados de forma a impedir possível hérnia interna.

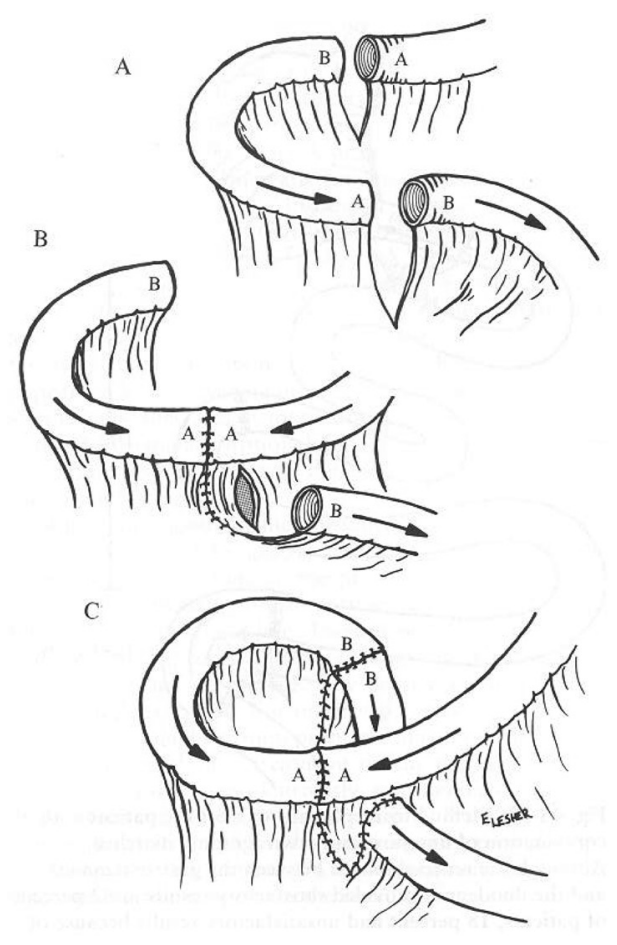

FIGURA 1 - Técnica de Rygick e Nasarov ${ }^{19}$ com a confecção de um segmento de alça anisoperistáltica

Na reintervenção, a alça intestinal estará dilatada, realiza-se então, a secção do segmento invertido da alça e sua reversão com posterior anastomose em plano único sero-muscular com fio PDS $® 3-0$ de forma a refazer o trânsito intestinal isoperistáltico.

$\mathrm{Na}$ sequência da técnica, iniciando o procedimento de STEP (Figura 2), a borda anti- mesentérica é marcada com caneta de forma a permitir a visualização do trajeto intestinal original dessa forma facilitando o posicionamento dos grampeadores. Perpendicularmente ao trajeto intestinal, eles são aplicados nas bordas anti-mesentérica e mesentérica, alternadamente, com $4,0 \mathrm{~cm}$ de extensão e $2,0 \mathrm{~cm}$ de distância entre eles. Não há necessidade de fechar o meso intestinal ${ }^{12}$ (Figura 3). Dessa forma, além do alongamento da alça, tem-se conformação em "degraus" fazendo com que o conteúdo intestinal assuma um circuito com sinuosidade mais longa e de menor calibre, possibilitando aos nutrientes maior tempo de contato com os sucos digestivos e com as vilosidades intestinais, tornando assim a absorção mais eficiente.

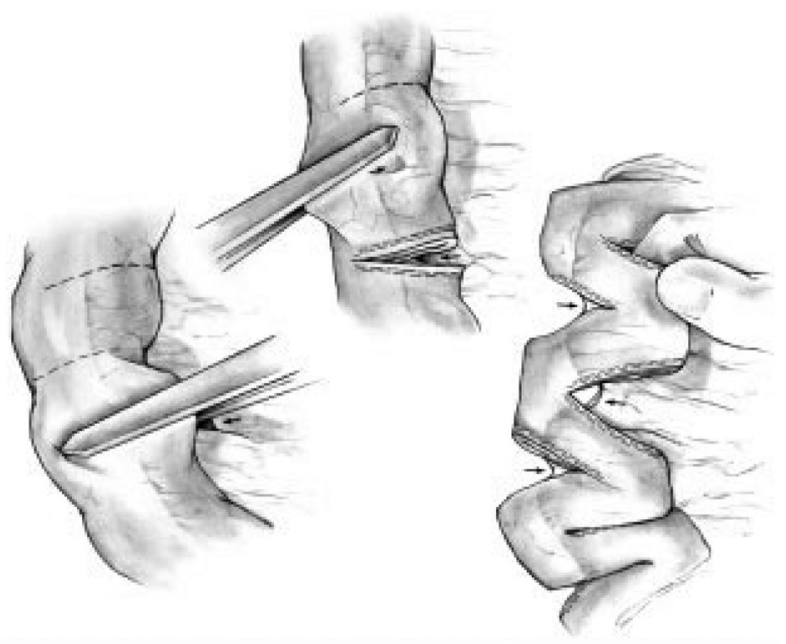

FIGURA 2 - No procedimento STEP - Serial Transverse Enteroplasty - os grampeadores lineares são aplicados perpedicularmente ao plano intestinal em direções alternadas. Um novo canal intestinal é assim formado, menor no diâmetro e maior em comprimento

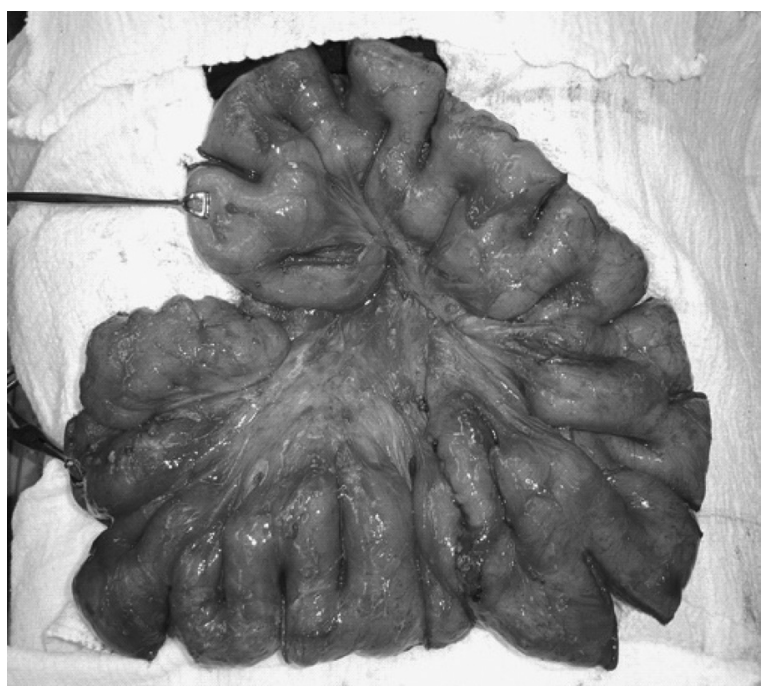

FIGURA 3 - Resultado final com aplicação de 25 grampeadores lineares de $75 \mathrm{~mm}$ na borda mesentérica e antimesentérica à STEP 
RESULTADOS

No caso descrito, a operação proposta possibilitou alongar a alça intestinal em 2,6 vezes do comprimento original, restabelecendo o peso corpóreo, a nutrição, reduzindo o número de exonerações de 15 para uma vez ao dia permitindo o retorno à vida social e conforto psicológico.

\section{DISCUSSÃO}

É difícil predizer o comprimento do intestino remanescente necessário para nutrição enteral adequada ${ }^{17}$. Os problemas metabólicos e sociais associados ao uso prolongado de nutrição parenteral total e a dificuldade de manter o acesso venoso central na SIC tornam o tratamento cirúrgico uma opção interessante.

O tratamento cirúrgico da SIC depende da idade do paciente, do comprimento do intestino remanescente, do diâmetro e da função intestinal ${ }^{24}$. Para reduzir o trânsito intestinal e aumentar o tempo de absorção existem várias técnicas consideradas, como a confecção de um segmento intestinal antiperistáltico, válvulas e interposição de segmento colônico 21,29,30. Também, o transplante intestinal é, atualmente, alternativa viável ao tratamento de pacientes com SIC ${ }^{7,18}$.

A confecção de um segmento intestinal antiperistáltico foi primeiramente descrito no século XX. Em estudo clássico na década de 5029, detalhouse técnica de reversão do segmento distal de intestino para reduzir o trânsito intestinal, aumentar a absorção e a taxa de sobrevivência em animais experimentais no modelo de SIC. Os autores observaram que um segmento longo de intestino invertido produzia obstrução intestinal nos animais ${ }^{29}$. Rygick e Nasarov ${ }^{19}$, em 1969, em estudo experimental utilizando cães, desenvolveram uma técnica de inversão de alça em que um segmento de $20 \mathrm{~cm}$ do íleo era dissecado, sendo utilizado como enxerto e anastomosado de forma a criar uma alça antiperistáltica ao longo do segmento intestinal. Posteriormente tiveram bons resultados no tratamento cirúrgico em humanos. Após esse estudo, a técnica foi utilizada por outros autores com resultados animadores relatados ${ }^{26}$.

Experiências clínicas com o uso de válvulas intestinais como a válvula de "nipple" (tipo mamilar) ainda são bem limitadas ${ }^{29}$ e podem causar obstrução intestinal e intussuscepção.

Outra alternativa para a correção do intestino curto é o alongamento de alça intestinal ${ }^{3,11,12}$. Doenças infecciosas crônicas, doença inflamatória intestinal e oclusão vascular intestinal crônica podem resultar em perda da função absortiva e peristáltica do intestino remanescente, sendo, dessa forma, contra-indicações ao alongamento intestinal ${ }^{17}$.

O tratamento cirúrgico mais empregado para aumentar a superfície absortiva intestinal é o alongamento longitudinal descrito por Bianchi $\mathrm{i}^{2,3}$, onde o comprimento do intestino delgado é duplicado. Essa técnicafoirealizadainúmerasvezes, predominantemente em crianças com intestino curto $17,20,27,32$. Outro método para alongamento intestinal, que duplica o segmento remanescente, foi descrito por Kimura ${ }^{11}$, técnica esta, limitada pela dificuldade de abordagem da cavidade abdominal.

O alongamento intestinal, pelas técnicas acima descritas, tem a vantagem de aumentar a função intestinal, corrigindo sua dilatação e peristalse inefetiva, aumentando o tempo de trânsito intestinal ${ }^{17}$, porém tem o risco potencial de fístula anastomótica e desvascularização. Por este motivo, evitada pelos autores.

O tratamento cirúrgico utilizando técnicas para promover a adaptação intestinal e o seu posterior alongamento, tem altas taxas de complicações e insucessos, porém são opções de menor complexidade e oferecem menores riscos que o transplante intestinal. Aproximadamente 700 transplantes intestinais foram realizados no mundo, sendo dois terços em populações pediátricas ${ }^{20}$. As indicações conhecidas para o transplante intestinal incluem o impedimento de nutrição parenteral por insuficiência hepática, trombose de veias centrais e frequentes crises de sepse por cateter central ${ }^{18,20}$. O emprego do transplante intestinal ainda é limitado pela taxa relativamente alta de complicações, como infecções, complicações cirúrgicas, rejeição aguda, doença enxerto-versus-hospedeiro e doenças linfoproliferativas pós-transplante ${ }^{7}$. Além disso, a sobrevivência de um e cinco anos pós-transplante é aproximadamente de $64 \%$ e $40 \%$, respectivamente ${ }^{20}$.

A operação de alongamento de alça intestinal pela técnica de STEP (Serial Transverse Enteroplasty) ${ }^{12}$ é baseada no princípio anatômico da irrigação sanguínea intestinal, tornando-se segura quando aplicado o grampeador linear perpendicularmente ao eixo longitudinal do intestino delgado. Quando o grampeador é aplicado seriadamente, como descrito por Kim, et al. ${ }^{12}$, cria-se um canal de intestino mais longo e estreito, apresentando resultado semelhante ao descrito por Bianchi ${ }^{3}$ e por Kimura ${ }^{11}$. Porém, o método de STEP tem vantagem de ser mais fácil, não apresentar anastomoses, o intestino não é aberto, o mesentério não é alterado, e o aumento total do comprimento intestinal dependente da dilatação prévia do intestino e do tamanho do canal criado ${ }^{23}$. Com o procedimento de Bianchi, a circunferência é reduzida pela metade, e com o procedimento STEP, o tamanho do canal e o seu diâmetro são determinados pelo cirurgião. Chang, et al. ${ }^{6}$ observou que o STEP melhora a manutenção do peso, nutrição e capacidade absortiva intestinal. A técnica de STEP já foi realizada com sucesso ${ }^{13} \mathrm{em}$ alguns pacientes como no caso descrito previamente pelos autores, apesar da longa espera em adquirir os grampeadores.

No descrito, o intestino remanescente era 
relativamente curto, $50 \mathrm{~cm}$ aproximadamente, não apresentando o ceco. Em adultos, a ressecção de $40-50 \%$ do total do comprimento intestinal é usualmente bem tolerada, porém, quando há a ressecção ileal com retirada da válvula íleo-cecal, pode haver desnutrição com menor grau de ressecção ${ }^{28}$. Isso ocorre porque essa válvula é importante barreira anti-refluxo de bactérias colônicas e ajuda a regulação da saída de fluidos e de nutrientes do intestino delgado ${ }^{28}$. A paciente evoluiu com SIC e desnutrição, beneficiandose, por esse motivo, da aplicação da técnica de Rygick e Nasarov ${ }^{19}$ aumentando o diâmetro do intestino delgado e possibilitando um posterior alongamento intestinal adequado pela técnica de STEP. Devido à dificuldade de manter o intestino delgado na posição adequada, a marcação da borda anti-mesentérica, no início da operação, com marcador de pele estéril, ajuda a manter a orientação ${ }^{13}$. Como observado no estudo de Kim, et al. ${ }^{13}$, não foram observadas hérnias internas no caso descrito, apesar de não ter-se fechado o defeito do mesentério criado pelo grampeador na utilização da técnica de STEP.

\section{CONCLUSÃO}

Essa técnica de inversão de $20 \mathrm{~cm}$ de alça intestinal e posterior reversão da alça associada à STEP - Serial Transverse Enteroplasty - é eficiente e surge como tratamento definitivo da SIC de forma promissora e segura. Apesar de requerer várias cargas de grampeadores, apresenta baixa morbidade e passa a tornar-se potencial substituto para o transplante intestinal.

\section{REFERÊNCIAS}

1. Andorsky DJ, Lund DP, Lillener CW, Jaksic T, DiCanzio J, Richardson DS, COller SB, Lo C, Duggan C. Nitritional and other postoperative management of neonates with short bowel syndrome correlates with clinical outcomes. J Pediatr. 2001; 139(1):27-33.

2. Bianchi A. Instestinal Lenghthening an experimenta and clinical review. J Roy Soc Med Suppl. 1984; 77(3):35-41.

3. Bianchi A. Intestinal loop lenghthening - A thechnique for icreasing small intestinal length. J Pediatr Surg. 1980; 15(2):14551.

4. Byrne TA, Parsinger RL, Young LS, Ziegler TR, Wilmore DW. A new treatment for patients with short-bowel syndrome. Ann Surg. 1995; 222(3):243-55.

5. Braun F, Broering D, Faendrich F. Small intestine transplantation today. Arch Surg. 2007; 392(3):227-38.

6. Chang RW, Javid PJ, Oh J, Andreoli S, Kim HB, Fauza D, Jaksic T. Serial transverse enteroplasty enhances intestinal function in a model of short bowel syndrome. Ann Surg. 2006; 243(2):223-8.

7. Cicalese L, Rastellini C, Sileri P, Abcarian H, Benedetti E. Segmental living related small bowel transplantation in adults. J Gastrointest Surg. 2001; 5(2):168-73.
8. Dudrick SJ, Latifi R, Fosnocht D. Management of the short bowel syndrome. Surg Clin North Am. 1991; 71(3):1991.

9. Galea MH, Holiday H, Carachi R, Kapila L. Short-bowel syndrome -A collective review. J Pediatr Surg. 1990; 27(5):592-96.

10. Huskisson LJ, Brereton RJ, Klely EM, Spitz L. Problems with intestinal lengthening. J Pediatr Surg. 1993; 28(5):720-22.

11. Kimura K. A new bowel elongation technique for the short-bowel segment Iowa models. J Pediatr Surg. 1993; 28(6):792-94.

12. Kim HB, Fauza D, Garza J, Oh J-T, Jacksic T. Serial transverse enteroplasty (STEP): a novel bowel lengthening procedure. J Pediatr Surg. 2003; 38(3): 425-29.

13. Kim HB, Lee PW, Garza J, Duggan C, Fauza D, Jacksic T. Serial transverse enteroplasthy for short bowel syndrome: a case report. J Pediatr Surg. 2003; 38(6):881-85.

14. Martin JR, Pattee CJ, Gardner C, Marien B. Massive resection of the small intestine. Can Med Assoc J. 1953;69(4):429-33.

15. Ninghtingale JMD. Management of patients with a short bowel. World J Gastroenterol. 2001; 7(6):741-51.

16. Platell CFE, C.J., McCauley RD, Hall JC. The management of patients with the short bowel syndrome. World J Gastroenterol. 2002; 8(1): 13-20.

17. Pokorny WJ, Fowler CL. Isoperistaltic Intestinal lengthening for short bowel syndrome. Surg Gynecol Obstet. 1991; 172(1):39-43.

18. Reyes J. Intestinal transplantation for children with short bowel syndrome. Semin Pediatr Surg. 2001; 10(2): 99-104.

19. Rygick AN, Nasarov LU. Antiperistaltic displacement of an ileal loop without twisting its mesentery. Dis Colon Rectum. 1969;12(6):40911.

20. Scoplapio JS. Treatment of short-bowel syndrome. Curr Opin Clin Nutr Met Care. 2001; 4 (6):557-60.

21. Shanbhogue LKR, Molennar JC. Short boeel syndrome: metabolic and surgical management. Br J Surg. 1994; 81(4)86-99.

22. Sondheimer JM, Cadnapaphornchal M, Sontag M, Zarbe GO. Predicting the duration of dependence on parenteral nutrition after neonatal intestinal resection. J Pediatr Surg. 1998; 132(1): 80-84.

23. Thompson JS. Comparison of massive repeated resection leading to short bowel syndrome. J Gastrointest Surg. 2000; 4(1):101-4.

24. Thompson JS, Langnas AN, Pinch LW, Kaufman S, Quigley EMM, Vanderhoof JA. Surgical approach to short bowel syndrome - en experience in population of 160 patients. Ann Surg. 1995; 222(4):600-5.

25. Thompson JS. Surgical considerations in the short bowel syndrome. Surg Gynecol Obstetr. 1993; 176(1):89-101.

26. Thompson JS, Langnas AN. Surgical approaches to improving intestinal function in the short bowel syndrome.Arch Surg.1999;134(7)706-9.

27. Thompson JS, Pinch LW, Murray N, Vanderhoof JA, Sclutz LR. Experience with intestinal lengthening for the short bowel syndrome. J Ped Surg. 1991; 26(6): 721-24.

28. Vanderhoof JA, Langnas AN. Short bowel syndrome in children and adults. Gastroenterology. 1997; 113(5):1707-78.

29. Varnon AH, Georgeson KE. Surgical Options for short bowel syndrome. Semin Pediatr Surg. 2001; 10(2):91-8.

30. Warmer BW, Chset MS. Nontransplant surgical options for management of the short bowel syndrome. J Pediatr Gastroenterol Nutr.1993;17(1):1-12.

31. Walters C, FM, Dejong CHC, Deutz NEP, Heineman E. Intestinal adaptation in short bowel syndrome. ANZ J Surg. 2002; 72(3):22936.

32. Weber TR. Isoperistaltic bowel lenghthening for short bowel syndrome in children. Am J Surg. 1999; 178(6):600-4.

33. Weser E. Clinical management of patients after small bowel resection. Gastroenterology. 1976; 71(1):146-50.

34. Wilmore DW, Robinson MK. Short bowel syndrome. World J Surg. 2000; 24(12): 1486-92. 